\title{
PATHWAY ANALYSIS IN PLANT CELLS
}

MIAMI-In contrast to the almost exponential growth of information about the pathways animal cells use to translate receptor activation into gene expression, signal transduction in plant cells has remained largely a series of black boxes. But at this year's Miami Bio/Technology Winter Symposium here, Nam-Hai Chua of Rockefeller University (New York) described recent results that cast the first light into one such box. Using an approach that recalls Charles Yanofsky's seminal experiments on tryptophan biosynthesis in Escherichia coli, Chua and his principal collaborator Gunther Neuhaus of the Swiss Federal Institute of Technology (Zurich) have begun to elucidate the nature and organization of the biochemical intermediates between the photoreceptor phytochrome and induction of the corresponding light-responsive genes.

The core of this approach is asking whether one can phenotypically revert mutants blocked at one stage of a pathway by providing a later intermediate. In this case, Chua and Neuhaus utilized the phytochrome-A-deficient aurea mutant of tomato, which was isolated almost ten years ago by $M$. Koornneef at the Agricultural University of Wageningen in the Netherlands. Lightgerminated aurea seedlings morphologically and biochemically resemble wildtype seedlings germinated in the dark. They have elongated hypocotyls, lack anthocyanin and chlorophyll, and do not develop chloroplasts. Since the last three properties are readily examined in single cells, microinjection provided an ideal means for chemically dissccting these pathways.

\section{Initial experiments}

In their first experiments, the researchers injected single hypocotyl cells of aurea with approximately 10,000 molecules of purified oat phytochrome A. These cells, but not their uninjected neighbors, subsequently displayed the pink pigmentation characteristic of anthocyanin and developed chloroplasts. Additionally, when a chimeric construct, containing the light-responsive promoter of the chlorophyll $a b$ binding gene $(c a b)$ fused to a betaglucoronidase (gus) reporter, was injected with the phytochrome, localized gus activity was detected upon exposure to a chromogenic substrate. Thus, not only is it possible to biochemically complement the phytochrome deficiency, but the signals between lightactivated phytochrome and anthocya- nin and chlorophyll biosynthesis appear to be cell autonomous.

With the basic system working and three assays in hand, the researchers were able to begin their pathway analysis in earnest. They first asked, reasonably enough, if phytochrome signaling involved calcium mobilization. Injection of phytochrome followed by incubation in the presence of calcium-channel blockers like verapamil gave a clear result. Cab-gus responsiveness and chloroplast development were elimated but the ability to synthesize anthocyanin was not. This suggests that activated phytochrome generates at least two sets of divergent signals. If those leading to cab-gus expression and chloroplast development proceed through elevating intracellular calcium levels, a good candidate for a further downstream effector might be calmodulin, which regulates a number of protein kinases by calcium binding. Consistent with this, when phytochrome-injected cells were exposed to calmodulin antagonists such as trifluroperazine, cab-gus expression and chloroplast development were again inhibited while anthocyanin synthesis remained unaffected. The logic of this kind of pathway analysis dictates that calcium and activated calmodulin should therefore be able to substitute for phytochrome A in the former reactions but not in those leading to an thocyanin production. And indeed this is exactly what the researchers found.

\section{Is light needed?}

With the ability to thus selectively induce light-responsive genes by simply injecting calcium, Chua and Neuhaus were able to address the fundamental but hitherto clusive question of whether light is required for more than the initial activation of phytochrome. To do this, they injected aurea hypocotyl cells with calcium under a green safe-light. After 24-48 hours of further incubation in the dark, they were able to demonstrate $c a b$-gus expression, implying that light is not necessary for the activation of this class of photo-responsive promoters beyond the calcium-mobilization stage of the signal-transduction pathway. Whether there are other lightsensitive steps between phytochrome activation and calcium mobilization will be answerable as the earlier intermediates are identified.

Indications that GTP-binding proteins are involved in these earlier stages were provided by using guanine nucleotide analogs. Injecting GDP-beta-S, an inhibitor of G-protein activation, along with phytochrome A blocked cab-gus expression, chloroplast development, and anthocyanin synthesis (although it is still unclear if all three reactions utilize the same G-factor). Conversely, the G-protein activator, GTP-gamma-S, could substitute for phytochrome $\mathrm{A}$ in two of the three reactions: Anthocyanin production and cab-gus responsivness could be detected, but chloroplast development did not occur, suggesting that the latter two pathways are also partially divergent.

Alfred Hershey once defined heaven as having a simple, reproducible experimental system that could be manipulated in increasingly sophisticated ways to answer and generate increasingly difficult questions. In the aureamicroinjection model, Chua and Neuhaus may have found their "Hershey heaven." -Harvey Bialy

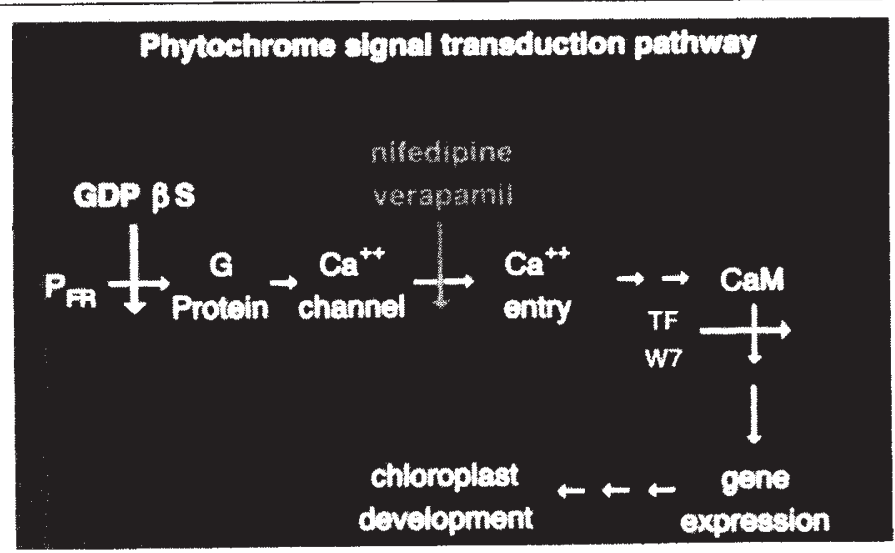

Introducing combinations of biochemical stimulators and inhibitors into single cells of a phytochrome-deficient mutant of tomato has produced the first outline of the pathway involved in transducing activation of this photoreceptor to multiple gene expression. 